\title{
Extracción y cuantificación de antocianinas a partir de los granos de Zea mays L. (maíz morado)
}

\section{Extraction and quantification of anthocyanins from the grain of Zea mays L. or purple corn}

\author{
Rosa Sotomayor ${ }^{1}$
}

http://dx.doi.org/10.21503/CienciayDesarrollo.2013.v16i1.06

\section{RESUMEN}

El presente trabajo de investigación evaluó la extracción y cuantificación de antocianinas a partir de los granos de Zea mays L. (maíz morado), mediante espectrofotometría UV -Visible.

Se obtuvo un pigmento natural en polvo del maíz morado Zea mays $L$. a nivel de laboratorio, con un rendimiento del 2, 25\% en extracto de $\mathrm{NaOH}$ para su aplicación en la industria alimentaria.

Palabras clave: pigmento natural Zea mays L, antocianinas, cuantificación.

\section{ABSTRACT}

The present research evaluated the extraction and quantification of anthocyanins from the grains of Zea mays L. (purple corn) by UV-visible spectrophotometry.

This gave a natural pigment of purple corn powder Zea mays L. a laboratory scale with a yield of 2 $25 \% \mathrm{NaOH}$ extract for use in the food industry.

Keywords: natural pigment Zea mays L, anthocyanins, quantification.

\section{INTRODUCCIÓN}

Del Zea mays L. o maíz morado, se obtiene un colorante púrpura usado en la industria farmacéutica y alimentaria como colorante natural. Estos pigmentos naturales presentes en el maíz también tienen aplicaciones medicinales como agentes anticancerosos, antioxidantes e hipocolesterolémicos. Los constituyentes benéficos son las antocianinas y los compuestos fenólicos, entre otros las antocianinas son colorantes na- turales, solubles en agua, presentes en muchas plantas. De acuerdo con la importancia de los colorantes naturales en el mercado mundial y la necesidad de obtenerlos en polvo, para facilitar su transporte y dosificación, con un tratamiento de secado bajo condiciones que no dañen el producto, se pretende aplicar una técnica a nivel de laboratorio para la obtención del colorante natural del maíz morado para luego ser utilizado en la industria alimentaria.

\footnotetext{
1 Magister Ciencia y Tecnología de los Alimentos. Docente Principal EAP Ingeniería Industrial UAP Filial Arequipa. e-mail: rosamsotomayor@yahoo.com
} 
El pigmento de antocianina tiene muchas acciones terapéuticas; entre ellas destacan, colesterol, cáncer de colon, combaten lo que es el stress oxidativo causado por los radicales libres ya que la antocianina es un potente antioxidante. El maíz morado, oriundo del Perú, tiene grandes cantidades del pigmento de antocianina.

\section{Hipótesis}

El extracto de antocianinas que se obtiene a partir de los granos de Zea mays L. (maíz morado), contendrían altas concentraciones de antocianina.

\section{MATERIALES Y MÉTODOS}

\section{Material}

Reactivos: agua destilada, $\mathrm{NaOH}$ al 2\%, ácido cítrico al 10\%, colorante sintético

Equipos: balanza analítica, cocina eléctrica, agitador magnético, espectrofotómetro UV visible, vaso de precipitado pequeños, pipetas de $10 \mathrm{ml}$, $5 \mathrm{ml} \mathrm{y} 1 \mathrm{ml}$, fiolas de $100 \mathrm{ml}$, tubos de ensayo, papel filtro, bagueta de vidrio, pizetas, gradillas para tubos de ensayo,vasos de precipitados de 100 y $250 \mathrm{ml}$ (pirex), matraz de $50 \mathrm{ml}$. mortero, pHmetro

\section{Método}

Análisis fitoquímico del maíz morado (Zea maiz)

Pesar 100 gr de maíz morado. Calentar $500 \mathrm{ml}$ de agua y antes de que llegue a ebullición colocar los 100 gr de maíz morado. Dejar por espacio de 20 minutos y retirar del fuego. Dejar enfriar. Realizar el análisis fitoquímico. Se procede a evaluar el extracto con las reacciones de Dragendorff que se debe cambiar a rojo a naranja en presencia de alcaloides; Shinoda debe cambiar a tonos rojo indicando la presencia de flavonoides, continuando con los ensayos que indica en la Tabla 1.

\section{Tabla 1. Estudio fotoquímico}

\begin{tabular}{|c|c|c|}
\hline MÉTODO & PROCEDIMIENTO & COLOR \\
\hline $\begin{array}{l}\text { Reacción de Dragendorff } \\
\text { (yoduro de bismuto y } \\
\text { potasio) }\end{array}$ & $\begin{array}{l}\text { a: } 8 \mathrm{~g} \mathrm{Bi}\left(\mathrm{NO}_{3}\right)_{3} .5 \mathrm{H}_{2} \mathrm{O} / 20 \mathrm{ml} \mathrm{HNO}_{3} \\
\text { b: } 27.2 \mathrm{~g} \mathrm{KI} / 50 \mathrm{ml} \mathrm{H}_{2} \mathrm{O} \\
\text { Mezclar, reposar, decantar supernadante. } \\
\text { Diluir a } 100 \mathrm{ml}\end{array}$ & Rojo a naranja \\
\hline Reacción de Shinoda & $\mathrm{Ms}+$ limadura $\mathrm{Mg}+\mathrm{HCl}$ conc. & Tonos rojo \\
\hline $\begin{array}{l}\text { Reacción de Tricloruro } \\
\text { férrico }\end{array}$ & $\begin{array}{l}\mathrm{R}: 1 \mathrm{~g} \mathrm{FeCl}_{3} / 100 \mathrm{ml} \mathrm{H}_{2} \mathrm{O} \\
\mathrm{Ms}+1 \mathrm{gt} \mathrm{R}\end{array}$ & Azul, verde negro \\
\hline Reacción de la gelatina & $\begin{array}{l}\text { R: } 1 \text { g gelatina/ } 100 \mathrm{ml} \mathrm{H}_{2} \mathrm{O}+10 \mathrm{~g} \mathrm{NaCl} \\
\text { Ms }+1 \text { gt } \mathrm{R}\end{array}$ & Precipitado \\
\hline $\begin{array}{l}\text { Reacción de Liebermann - } \\
\text { Burchard }\end{array}$ & $\begin{array}{l}1 \mathrm{mg} \mathrm{Ms} / \text { pocas gotas Anhídrido acético }+ \\
3 \mathrm{ml} \mathrm{H}_{2} \mathrm{SO}_{4} \text { conc } / 3 \mathrm{ml} \text { etanol }\end{array}$ & $\begin{array}{l}\text { Verde, azul verdoso (vía } \\
\text { rojo o azul) }\end{array}$ \\
\hline Reacción de la Ninhidrina & Ms + Ninhidrina & Pigmento púrpura \\
\hline Reacción de Molish & Ms $2 \mathrm{ml}+2 \mathrm{gt} \alpha$ naftol $1 \% / \mathrm{H}_{2} \mathrm{SO}_{4}$ conc & $\begin{array}{l}\text { Formación de un anillo } \\
\text { color oscuro }\end{array}$ \\
\hline
\end{tabular}

Fuente: Klukinski Claudia, Farmacognosia - $5^{\text {ta }}$ edición 


\section{Recolección y extracción del colorante}

Seleccionar los granos de maíz morado (Zea mays) del mercado San Camilo en la ciudad de Arequipa (Arequipa, Perú).

Se procedió a pesar 200 gr y someter a molienda y tamizado malla 100 , agregar $\mathrm{NaOH} 2 \%$ en un volumen de $300 \mathrm{ml}$ por un espacio de 12-16 horas. Separar la suspensión coloreada de las semillas.

Medir $\mathrm{pH}$ y agregar ácido cítrico al 10\% para disminuir el $\mathrm{pH}$ hasta alcanzar un $\mathrm{pH}$ de 2-2,5 y favorecer la precipitación del colorante. Filtrar al vacío, secar en el horno a $57^{\circ}$. Someter a molienda la pasta colorante. Pesar el colorante en polvo.

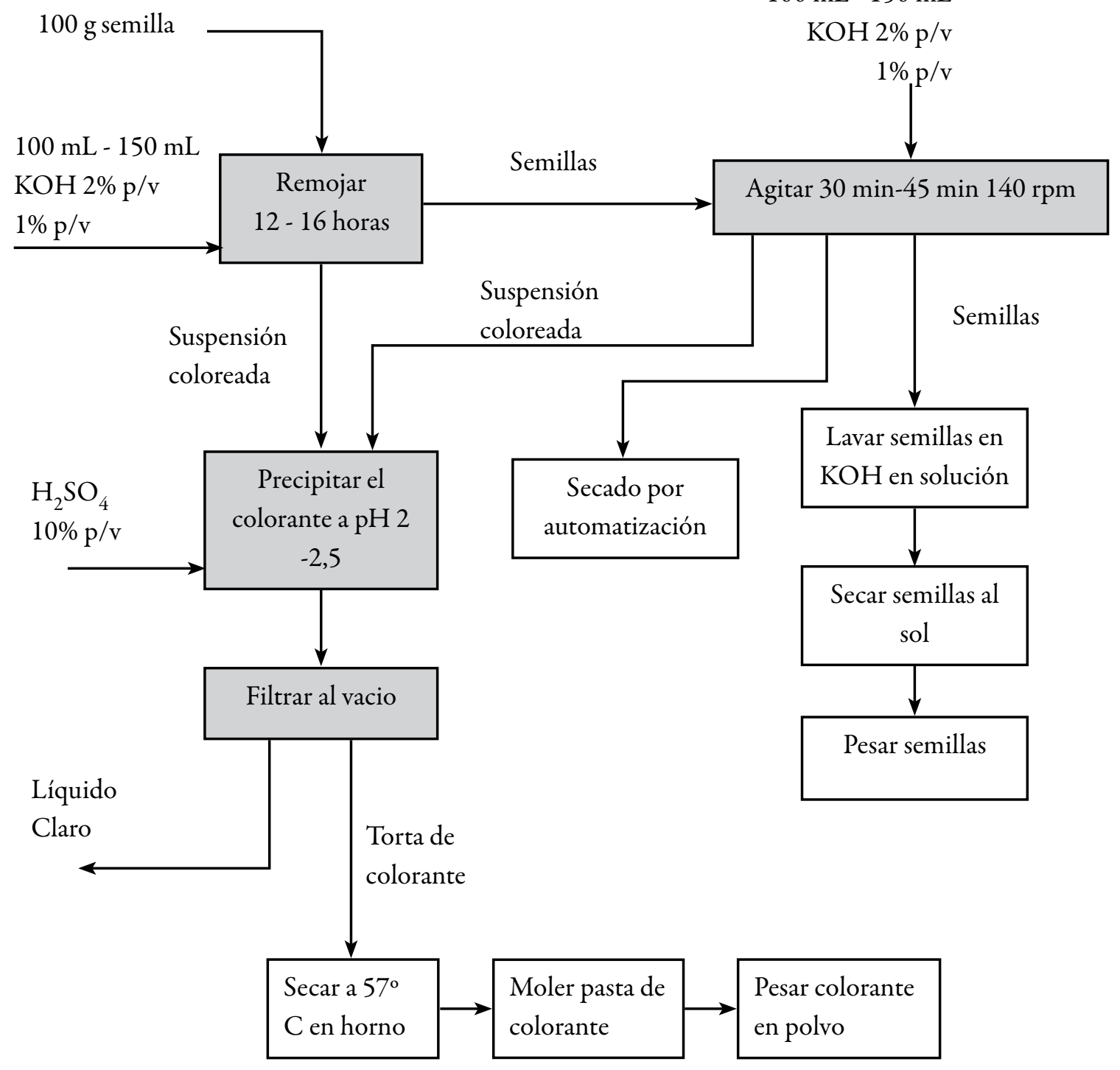

$100 \mathrm{~mL}-150 \mathrm{~mL}$

Figura1. Procedimiento para la extración del colorante

Fuente: Universidad de Colombia - grupo de investigación desarrollo y diseño de procesos -Departamento de Ingeniería de Procesos 
Se hizo dos ensayos donde se obtuvieron 4,5 y 3,5 mg de colorante respectivamente, de cada 200 g de maíz morado.

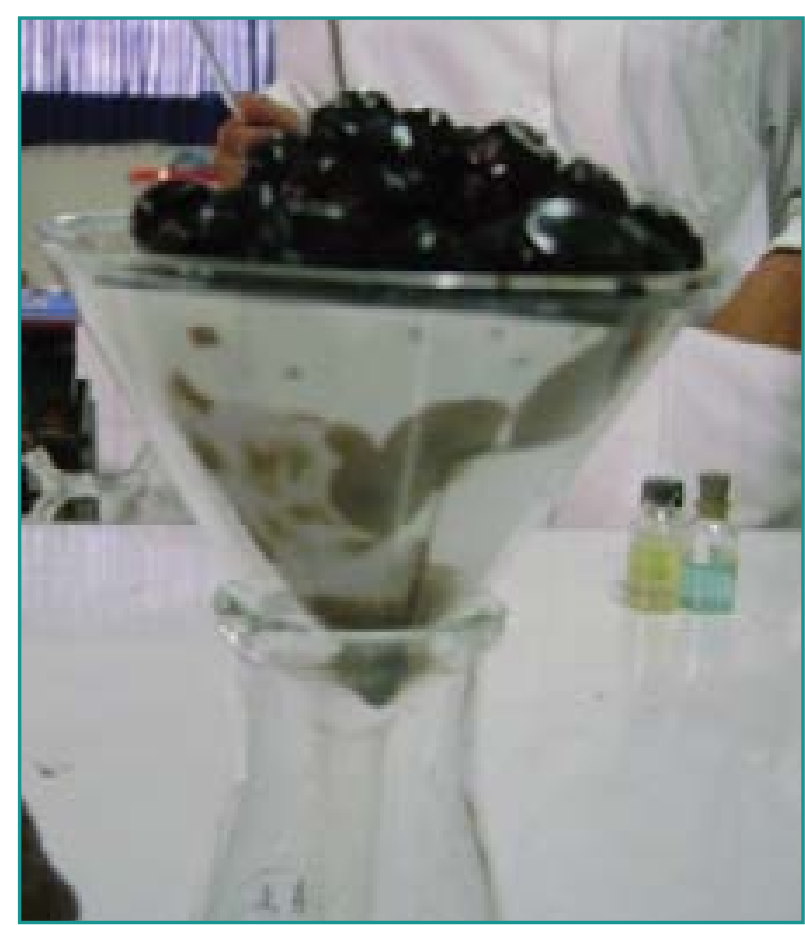

Figura 2. Filtración del extracto de semillas de maíz morado con $\mathrm{NaOH}$

Fuente: Elaboración propia

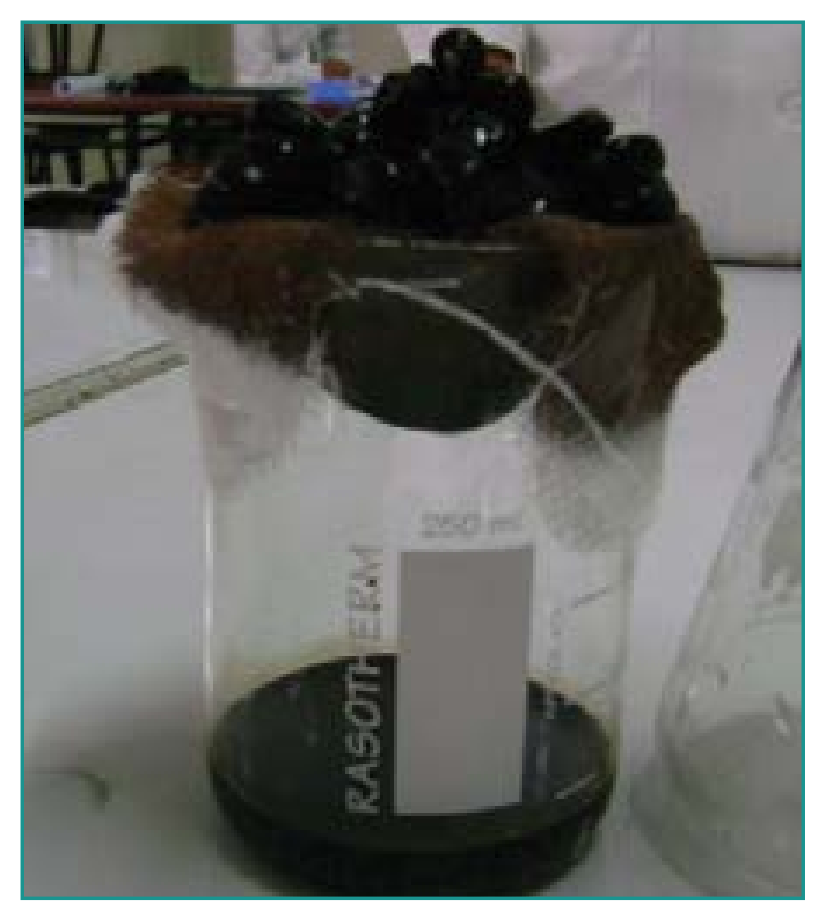

Figura 3. Filtración del extracto alcalino

Fuente: Elaboración propia

\section{Análisis instrumental de antocianinas}

Se determinó la concentración de antocianinas, en el espectrofotómetro UV visible a $510 \mathrm{~nm}$.

\section{RESULTADOS}

Los resultados obtenidos son los siguientes:

Tabla 2. Análisis fitoquímico del Maíz morado (Zea maiz. L.)

\begin{tabular}{ccc}
\hline Prueba de caracterización & Metabolito secundario & Resultado \\
\hline Reacción de Dragendorff & Alcaloides & - \\
\hline Reacción de Shinoda & Flavonoides & +++ \\
\hline Reacción de Tricloruro férrico & Compuestos Fenolicos & +++ \\
Reacción de la gelatina & Taninos & ++ \\
\hline Reacción de Liebermann & Esteroides o terpenos & - \\
Burchard & Aminoácidos libres & - \\
\hline Reacción de la Ninhidrina & Glicosidos & +++ \\
\hline Reacción de Molish & & \\
\hline
\end{tabular}

Fuente: Elaboración propia 


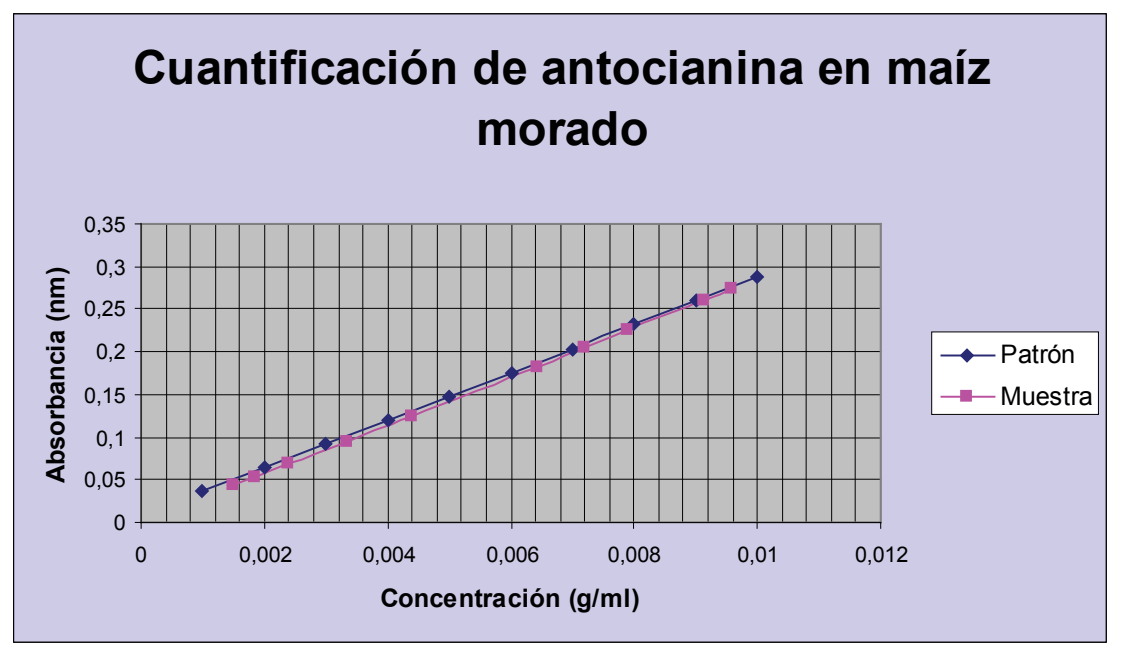

Figura 4 Fuente: Elaboración propia

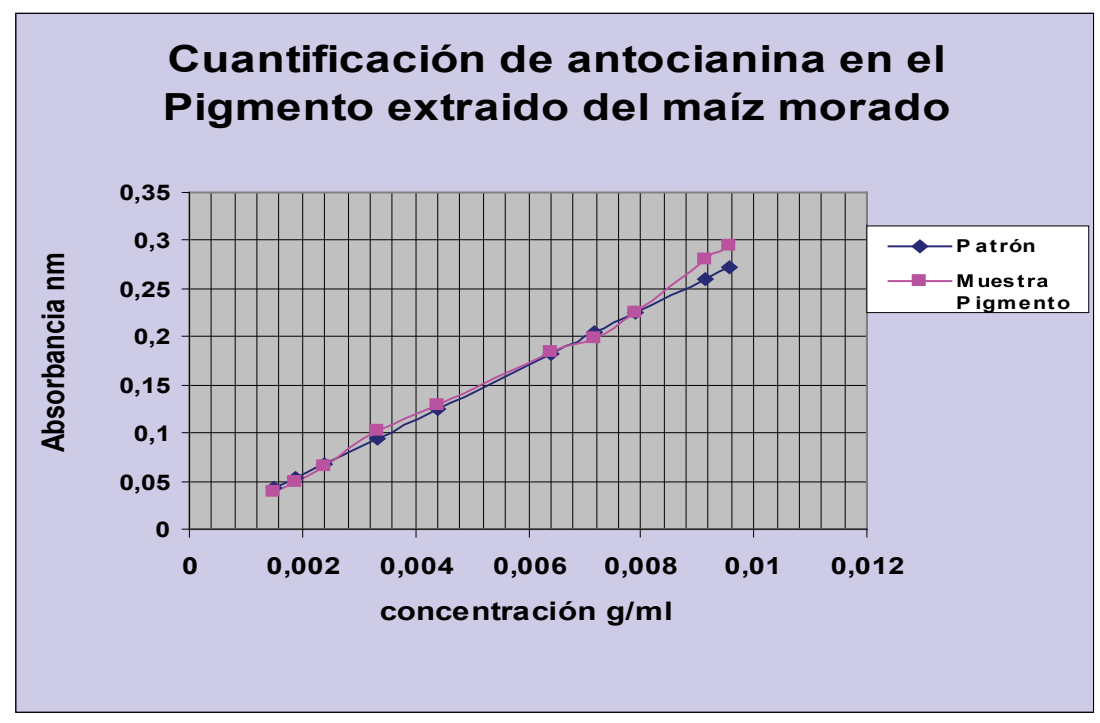

Figura 5 Fuente: Elaboración propia

\section{DISCUSIÓN}

Mediante el análisis fitoquímico se pudo determinar cualitativamente la presencia de flavonoides, compuestos fenólicos, glicósidos y taninos. Resultado positivo para estos metabolitos y negativo para alcaloides y los esteroides por lo que este extracto de maíz morado contiene mayor cantidad de compuestos fenolicos y glicosidicos que le otorgan sus propiedades terapéuticas.

En la Figura 4 la curva de calibración, en comparación con la curva del colorante sintético (096978), nos indica el estrecho acercamiento de las dos curvas de calibración indicando que los niveles o contenido de antocianina es alto. 
En la figura 5, la curva de calibración del pigmento obtenido del maíz morado haciendo una comparación con la curva del colorante sintético (096978), nos indica alto contenido de presencia de antocianinas en el pigmento en polvo obtenido.

\section{CONCLUSIONES}

Por medio del análisis fitoquímico se encontró la presencia de flavonoides, compuestos fenólicos, glicósidos y taninos, los cuales fueron identificados por los cambios de coloración frente a los diferentes reactivos.

La curva de calibración elaborada puede ser utilizada como curva patrón para determinar cuantitativamente la presencia de antocianinas en otras muestras.

De acuerdo a la determinación cuantitativa de la concentración de antocianina se pudo comprobar que el extracto de maíz morado (Zea maiz. L) contiene altas concentraciones de antocianinas.

Se logró obtener el pigmento natural, por procesos de ensayos a nivel de laboratorio, obteniéndose un rendimiento de $2,25 \%$ del pigmento natural en cada extracción.

\section{BIBLIOGRAFÍA}

1. Escobar Arango, Jaime. Pulverización de colorantes naturales por secado por atomización -Universidad de Colombia -grupo de investigación desarrollo y diseño de procesos -Departamento de Ingeniería de Procesos, Grupo de Investigación.jdevia@ea.t.edu.co.

2. Alzate, Marcela y Sabogal, Juan José. (2005). Pulverización de colorantes naturales a escala industrial. Proyecto de Grado de Ingeniería de Procesos. Medellín: Universidad EAFIT.
3. Collazos, C. 1996. Tablas Peruanas de Composición de Alimentos.

4. Brack-Egg, Antonio. 1999. Diccionario Enciclopédico de Plantas Útiles del Perú. Cusco, Perú: Imprenta del Centro Bartolomé de las Casas.

Correspondencia: Rosa Sotomayor

e-mail: rosamsotomayor@yahoo.com 